\title{
Effects of Two Warm-Up Modalities on Short-Term Maximal Performance in Soccer Players: Didactic Modeling
}

\author{
Chameseddine Guinoubi1,2, Hajer Sahli², Rim Mekni' ${ }^{3}$, Salma Abedelmalek ${ }^{4,5}$, \\ Karim Chamari ${ }^{1}$ \\ ${ }^{1}$ Research Laboratory "Sport Performance Optimization", National Center of Medicine and Sciences in Sport \\ (CNMSS), Tunis, Tunisia \\ ${ }^{2}$ Research Unit, High Institute of Sport and Physical Education, University of Jendouba, El Kef, Tunisia \\ ${ }^{3}$ Higher Institute of Education and Training Education, Tunis, Tunisia \\ ${ }^{4}$ Research Unit, High Institute of Sport and Physical Education, University of Sfax, Sfax, Tunisia \\ ${ }^{5}$ Department of Physiology, Faculty of Medicine, University of Sousse, Sousse, Tunisia \\ Email: makwiss@yahoo.fr
}

Received 6 February 2015; accepted 24 February 2015; published 27 February 2015

Copyright (C) 2015 by authors and Scientific Research Publishing Inc.

This work is licensed under the Creative Commons Attribution International License (CC BY).

http://creativecommons.org/licenses/by/4.0/

c) (i) Open Access

\begin{abstract}
The purpose of this study was to investigate the effects of two warm-up modalities (scheduled before a soccer match) on short-term maximal performance and physiological responses in soccer players. Twenty soccer players (Age $=22.1 \pm 4.4$ years; Height $=183.2 \pm 7.1 \mathrm{~cm}$, Body $\operatorname{mass}=77.6 \pm$ $8.5 \mathrm{~kg}$ ) participated in this study. They randomly performed two different pre-match warm-up protocols on separate days with different excitation order component sequences where the last component of the warm-up excitation sequence was imputed completed either in the middle (WU-1), or at the end of the warm-up (WU-2). During the completion of the warm-up, heart rate (HR), and ratings of perceived exertion (RPE) were recorded. After the warm-up, participants performed a repeated sprint ability test (RSA) and the five jump (5JT) test. The results revealed no significant differences in WU-1 and the WU-2 for HR and RPE. In WU-2 condition, significant differences in the 7 th acceleration phase of the repeated $10 \mathrm{~m}$ sprint $(\mathrm{S} 7)(p<0.05)$ was observed in comparison to WU-1. Likewise, the performance of 5JT $(p<0.05)$, and the $10-\mathrm{m}$ of the RSA $(p<0.05)$ increased to WU-2 in comparison with WU-1. In conclusion, we recommended performing warm-up at the end of pre-match rather than in the middle to ameliorate the performance of soccer players during the competition.
\end{abstract}

\section{Keywords}

Warm-Up, Sprint, Jump, Soccer Players, Didactic, Modeling

How to cite this paper: Guinoubi, C., Sahli, H., Mekni, R., Abedelmalek, S., \& Chamari, K. (2015). Effects of Two Warm-Up Modalities on Short-Term Maximal Performance in Soccer Players: Didactic Modeling. Advances in Physical Education, 5, 70-76. http://dx.doi.org/10.4236/ape.2015.51009 


\section{Introduction}

Warm-up is considered an essential factor to prevent athletes from muscular injury and to improve performance (Gracielle et al., 2007). The warm-up period is defined as a preparatory exercise designated to enhance subsequent competition or training performance (Hedrick, 1992). The purpose of warm-up is to prepare the athlete physiologically and psychologically, and to reduce the risk of injury (Bourne, 1992).

Several studies were conducted in the 1950s-1970s to investigate the effects of warming-up on athletic performance (Richards, 1968). In this context, approximately $60 \%$ of these studies found that warm-up was better to perform than no warm-up, whereas $\sim 11 \%$ found that no warm-up was better, and the remaining $\sim 29 \%$ found no significant differences between different protocols of warm-up and no warm-up (Blank, 1955).

Soccer requires high-intensity intermittent efforts (Fradkin, 2002) that include many sprints of different durations, rapid accelerations and decelerations, jumping, agility, etc. Thus, it seems that soccer needs an active warm-up. In this context, previous studies have shown that active warm-up increases performance (Ekblom, 1986). Most active warm-up routines integrate four types of exercise: "cardiovascular" often based on running; "muscular" including some "explosive strength" exercises; passive or active "stretching"; "specific" (i.e., miming the characteristics of the sport exercise). The specificity of these exercises is very important and most of them are miming some aspects (e.g., posture, rhythm, muscular action, inter-limb coordination, and angular displacement) of the competitive sport exercise or a part of it.

There is little data in the literature supporting that warm-up is a desirable part of pre-competition preparation. Consequently, there is more equivocation over what type of stretch, if any, to apply as part of warm-up strategies. In this context, the optimal warm-up modalities are still controversial (Bishop, 2003). For example, vertical jumping ability has been shown to be improved following strength-related warm-up (Brown et al., 2008; Woods et al., 2007; Burkett et al., 2005), or deteriorated if warm-up includes some static stretching (Gourgoulis 2003; Saez Saez et al., 2007).

Furthermore, the assessment of soccer players' sprint performance was well documented in the last decade. Previous studies measured soccer players' 30-m sprint performance with 2- to 3-minute recovery in-between attempts (Holt \& Lambourne, 2008; Young \& Behm, 2003). However, during a 90-minute game, it is unlikely for a player to have 2 to 3 minutes of passive recovery between sprints. He actually performs sprints and repeats them with a short recovery time, i.e., repeated-sprint ability (RSA) (Chamari et al., 2004). Therefore, RSA is considered to more effectively simulate and reproduce soccer game performance as compared with a single bout of sprint (Wisloff et al., 2004; Spencer et al., 2005). In this context, Rampinini et al. (2009) have shown that RSA performance is related to soccer game sprint performance and that soccer coaches have implemented a series of short sprints, in order to prepare their players for the coming match performance. In that regard, RSA tests consisting of less than 10 sprint bouts with 20- to 30-second recovery have been recommended (Stolen et al., 2005). Contrary to a single sprint in which energy is mainly derived from anaerobic metabolism, the energy during RSA (sprints and recovery) is derived from both anaerobic and aerobic metabolisms (Fitzsimons et al., 1993).

Based on the facts that warming-up in soccer should include all components requested during games, and that knowing that warming-up modalities are controversial, the purpose of the present study is, therefore, to investigate the effect of sprint sequence placed at the end or at the middle of the pre-match warm-up on short-term performances in soccer players.

\section{Methods}

\subsection{Subjects}

Twenty male soccer players voluntarily participated in the study. The subjects were engaged in soccer for at least 6 years. Descriptive characteristics of the participants are presented in Table 1. They trained for at least four sessions per week in addition to one official game match per-week. The training sessions lasted 90 min approximately. All subjects were examined by the team physician prior to the study, with a particular focus on orthopedic and other conditions that might preclude resistance training. All procedures were approved by the Institutional Review local Committee for the ethical use of human subjects, according to current national laws and regulations. Participants gave written informed consent after receiving both a verbal and a written explanation of the experimental design and its potential risks. 
Table 1. The heart rate (HR) and rate of perceived exertion (RPE) scores revealed after the two warm-up modalities (i.e., in the middle (WU-1) and the end (WU-2) of the competition).

\begin{tabular}{cccc}
\hline & WU-1 & WU-2 & $p$ \\
\hline RPE & $3.05 \pm 0.39$ & $3.01 \pm 0.32$ & NS $(p=0.06)$ \\
HR (Batt/min) & $143.70 \pm 15.49$ & $143.30 \pm 11.81$ & NS $(p=0.91)$
\end{tabular}

*NS: no significant difference between WU-1 and WU-2.

\subsection{Experimental Design}

In a randomized order, the players performed a five-jump test and a RSA test after two warm-up protocols. The first warm-up protocol (WU-1) started with a 4-minute low-intensity jog followed by 8 minutes of general part (composed by moderate exercises of upper limbs, moderate exercises of lower limbs, and dynamic stretching), followed by 5 -minute sprint exercises before performing 8 minutes of specific warm-up sequence consisting of exercises with ball. The second warm-up protocol (WU-2) was performed similarly to the first one. However, the sprint sequence was placed at the end of warm-up (after specific sequence). 10 minutes after each warm-up protocol, subjects performed a five-jump test and then a RSA test with a 2-minute passive recovery between tests. After each test physiological responses data were retained (i.e., HR and RPE).

\subsection{The 5-Jump Test (5JT)}

The 5JT test is a practical, validate and alternative to estimate lower limb explosive power of selected population (Chamari et al., 2004). The 5JT consists of 5 consecutive strides with joined feet position at the start and at the end of the jumps. The subject attempts to cover the greatest horizontal distance possible by performing a series of five forward jumps with alternate left and right foot contacts. Subjects were given three trials; the best result (greatest distance) was obtained for analysis.

\subsection{The Repeated-Sprint Ability Test (RSA)}

The RSA test consisted of seven 30 -m sprints $(7 \times 30 \mathrm{~m})$ separated by a 25 -second passive recovery period (Spencer et al., 2005). This test was adapted from previous RSA cycling and running tests (Impellizzeri et al., 2008) that have been shown to be reliable and valid (Impellizzeri et al., 2008). A hand-held stopwatch measured the time for the 25 -second recovery. The sprint times were recorded by electronic speed time system (Brower Timing Systems, Salt Lake City, UT, USA,) placed at departure, $10 \mathrm{~m}$ and $30 \mathrm{~m}$. The players stood $0.5 \mathrm{~m}$ behind the sensor before they started sprint. Each player was instructed and verbally encouraged to give a maximal effort during all tests, The timing gate at sprint 30-m can be divided into two phases: the acceleration phase $(0-10$ $\mathrm{m}$ ) and time spent in the maximal-velocity phase was calculated by subtracting the acceleration time from the overall time. The reliability of the RSA test was established in a previous study (intraclass correlation coefficient $[\mathrm{ICC}]=0.98, p<0.0001$ with a coefficient of variation $[\mathrm{CV}]$ of 3.6\%) (Spencer et al., 2005). The ICC and CV for the total time (TT) were 0.95 and $3.4 \%$, respectively $(n=20)$.

\subsection{Training Intervention}

Study procedures took place in the university, on a football field in the morning and on 2 nonconsecutive days. Each group performed randomly two different warm-up protocols: WU-1 consisted of general warm-up, sprint sequence and finally, specific warm-up; the WU-2 including general warm-up, specific warm-up and a sprint. After 10 min of passive rest, the physical tests (5JT and RSA) were completed done.

The general warm-up consisted of, common for all, of jogging for 4 minutes and up to including. 5 exercises to solicit the upper limbs (rotation, balancing as well as elevation and depression, done with a light jog to and fro over a distance of $20 \mathrm{~m}$ ), followed by 5 exercises soliciting the lower limbs (elevation movement knee, heel to buttocks elevated, lateral displacement of both side and finally a rear run, every works runs with slightly pace). This sequence was completed with a series of lower body dynamic stretches; the movements selected were intended to warm-up the muscles used in soccer game. They mimic functional movements found in a competitive match, and the movements were carried out while jogging. 
The specific warm-up lasted 8 minutes and consisted of the same exercise done with balls achieve with some working in pairs, exchange of different kinds of passes with both inside feet, shot with both feet, a long pass after a control, centering, hits, fly passes, head kicks. This specific sequence was finished by an opposition of ball conservation ( 5 against 5 , on pa pitch measured $20 \mathrm{~m} \times 30 \mathrm{~m}$ ) limited to two touches ball.

Subjects completed a 4 minutes jog-run general warm-up before being assigned to either WP-1, or WP-2 warm-up conditions. In the WP-1 condition, subjects underwent 8 min of upper, and lower body active dynamic stretching (ADS) before completing 8 minutes of soccer-specific warm-up (SWP).

Each participant's heart rate (HR) and rate of perceived exertion (RPE) on the scale of (Impellizzeri et al., 2008) were collected in Table 2.

\subsection{Statistical Analysis}

All statistical tests were processed using STATISTICA Software (Stat Soft, USA). The Kolmogorov-Smirnov test of normality revealed the data were normally distributed. Once the assumption of normality was confirmed, parametric tests were performed. Performance measures were analysed using one way ANOVA with repeatedmeasures. The reliability of the RSA test was analyzed using the intra-class correlation coefficient [ICC]. The level of statistical significance was set at $p<0.05$.

\section{Results}

The statistical analysis revealed that physiological parameters (i.e., RPE and HR) were unaffected after the two warm-up protocols (i.e., WU-1 and WU-2) $(p<0.05)$. Likewise, our results showed that performance of the five jump test increased significantly $(p<0.05)$ after the WU-2 with ICC for this analysis $(\sim 0.87)$. Our results showed a significant improvement in the performance of sprint of $10 \mathrm{~m}$ (i.e., 7 th sprint) and $20 \mathrm{~m}$ (i.e., 1st sprint) after the WU-2 $(p<0.05)$ in comparison with WU-1. Concerning the results of repeated sprint of $30 \mathrm{~m}$, significant differences were observed in 1st, 2nd and 7th sprint $(p<0.05)$ with better performance after WU-2.

\section{Discussion}

The aim of the present study was to investigate the effect of including a sprint sequence in the end or at the middle of the pre-match warm-up on physical performance in soccer players. Our results showed that the inclusion of sprint sequence would be more beneficial for performance when it is placed at the end of warm-up. However, the soccer players were able to better jumping and sprinting and thus to develop optimal performance.

The major finding of our study was that performances of pre-game soccer warm-up including sprint sequence at the end of warm-up were better than a sprint sequence placed in the middle of warm-up. So, we found that the charge load imposed by the two warm-up protocols was similar and no significant difference $(p<0.05)$ was found for either RPE or HR. Previously, it has been demonstrated that performing a warm-up before any activity enhances performance during competition (Fradkin et al., 2010). Soccer had several demands such as physical,

\section{Table 2. Performance results.}

\begin{tabular}{|c|c|c|c|}
\hline Variables & WU-1 & WU-2 & $\mathrm{P}$ \\
\hline $5 \mathrm{JT}(\mathrm{m})$ & $11.51 \pm 0.32$ & $11.88 \pm 0.41$ & $0.03^{*}$ \\
\hline RSA $10 \mathrm{~m}$ & $2.02 \pm 0.09$ & $1.95 \pm 0.09$ & $0.05^{*}$ \\
\hline $\mathrm{S} 110 \mathrm{~m}$ & $2.00 \pm 0.10$ & $1.91 \pm 0.14$ & 0.12 \\
\hline $\mathrm{S} 210 \mathrm{~m}$ & $1.97 \pm 0.15$ & $1.91 \pm 0.13$ & 0.37 \\
\hline $\mathrm{S} 710 \mathrm{~m}$ & $2.00 \pm 0.14$ & $1.89 \pm 0.06$ & $0.05^{*}$ \\
\hline $\mathrm{S} 120 \mathrm{~m}$ & $2.59 \pm 0.12$ & $2.49 \pm 0.16$ & $0.05^{*}$ \\
\hline $\mathrm{S} 720 \mathrm{~m}$ & $2.66 \pm 0.18$ & $2.72 \pm 0.13$ & 0.25 \\
\hline RSA $30 \mathrm{~m}$ & $4.66 \pm 0.15$ & $4.63 \pm 0.21$ & 0.26 \\
\hline $\mathrm{S} 130 \mathrm{~m}$ & $4.56 \pm 0.14$ & $4.41 \pm 0.21$ & $0.05^{*}$ \\
\hline $\mathrm{S} 230 \mathrm{~m}$ & $4.56 \pm 0.15$ & $4.41 \pm 0.21$ & $0.05^{*}$ \\
\hline $\mathrm{S} 730 \mathrm{~m}$ & $4.71 \pm 0.18$ & $4.54 \pm 0.16$ & $0.03^{*}$ \\
\hline
\end{tabular}

5JT: five jump test; RSA: repeated sprint ability; $10 \mathrm{~m}$ : acceleration phase; $20 \mathrm{~m}$ : maximal velocity phase; $30 \mathrm{~m}:$ continuous sprint; ${ }^{*} p<0.05$. 
technical and tactical. However, the warm-up is an important component of physical exigencies to prepare player for match.

The results showed that the acceleration phase of the seventh sprint of the RSA test was significantly better after the WU-end than after WU-mid.

This finding supports the study of (Sayers et al., 2008) who have found that jog $800 \mathrm{~m}$, forward skips $4 \times 30$ $\mathrm{m}$, side shuffles $4 \times 30 \mathrm{~m}$, and backward skips $4 \times 30 \mathrm{~m}$ wish can be considered like dynamic warm-up with excitation exercise improve acceleration phase. In the same context, Little \& Williams (2006) used 3 warm-ups protocols ending with $\sim 4$ minutes of incremental intermittent sprint and agility runs. They found that the best result in 10-m acceleration is observed with dynamic stretching, which support our finding since we have used dynamic stretching at the end of the general sequence of the two warm-ups. This result is also supported by the fact that the RSA test of 10-m calculated separately had a significant difference $(p<0.05)$ between WU-1 and WU-2. Additionally, performance obtained during the 5JT is significantly better $(p<0.05)$ in WU-2 than WU-1. One of the possible mechanisms behind the enhanced jumping and sprinting performance after dynamic-style warm-up is Post-Activation Potentiation. Indeed, it has been shown that activation of a muscle, may cause an enhanced of performance for some time after the cessation of the activation (Sale, 2002). PAP may be a result of increased phosphorylation of myosin light chains, increasing the calcium sensitivity of the myofilaments (Sale, 2002).

In the first 20-m high speed the WU-2 protocol produced significantly faster $(p<0.05)$ runs than did the WU1 protocol, no significant result was found in the other six $20-\mathrm{m}$ of the successive RSA-test sprints, this may by explained with the fact that, maximum stride length only occurs when the runner has reached peak speed that is, during the maximal-speed phase, additionally during it both stride length and the stretch shortening cycle duration are at their respective greatest, in the present study the emplacement of excitation sequence at the end (WU-2) may increase this effect which observed only in the beginning in the study of (Sayers et al., 2008).

The findings of this study are establish in $30 \mathrm{~m}$ sprinting WU-2 have significant better results for the first, second and the last $30 \mathrm{~m}$ among the seven sprints of the RSA protocol then WU- 1 . This result confirms those in previous studies showing that dynamic exercise increases the power and speed performance (Faigenbaum et al., 2005; Faigenbaum et al., 2006). In fact, Gelen (2010) found that dynamic exercises performed after 5 minutes of jogging positively affect sprint, slalom dribbling, and penalty kick performances and thereby power performance. Faigenbaum et al. (2006) investigated the acute effects of different warm-up protocols on anaerobic performances in teenage athletes. They stated that dynamic warm-up practice positively affect sprint, medicine-ball toss, and vertical jump performance.

Faigenbaum et al. (2005) evaluated the acute effects of different warm-up protocols on fitness performances. Or investigation demonstrated that moderate to high-intensity dynamic warm-up exercises activate high power performance.

\section{Practical Applications}

The results of our study, including the sprint activity sequence at the end of warm-up could improve running speed during a 30-m sprint, $20-\mathrm{m}$ maximal-speed, $10-\mathrm{m}$ acceleration and $5 \mathrm{JT}$ performance. Soccer success can be dependent on maximal performance which we are aware of the potential positive effects of the insert of sprint sequence at the end of any pre-competitive warm-up. This finding is of great practical interest for coaches soccer and fitness coach trainers.

\section{Conclusion}

Previous investigators have suggested that a warm-up including dynamic exercises that replicate movements to be performed during the activity are beneficial to performance.

Given the originality of this study, success can be dependent on maximal performance, which is aware of the potential positive effects of the insert excitation part at the end of any pre-competitive warm-up. This finding is of great practical interest for coaches and fitness training. In the end, we recommended that the pre-match warm-up should end with excitation sequence based on sprints. Although more researches in the future are needed on this topic, it can be suggested that dynamic exercise performed for warm-up purposes may increase explosive-power production increasing neuromuscular functionality. It can be proposed that the excitation sequence at the end of warm-up increases the excitability of speed-contracted units of target muscles, thereby making these units ready to play an important role during certain activities such as sprinting performance and 
jumping abilities.

\section{Acknowledgements}

This study was supported by the Ministry of Higher Education and Scientific Research, Tunisia. We are grateful to all of the players who participated so willingly in the study.

\section{References}

Bishop, D. (2003). Warm-Up II. Performance Changes Following Active Warm-Up and How to Structure the Warm-Up. Sports Medicine, 33, 483-498. http://dx.doi.org/10.2165/00007256-200333070-00002

Blank, L. B. (1955). Effects of Warm-Up on Speed. Athletic Journal, 35, 10.

Bourne, G. (1992). The Physiological Basis of the Warm-Up. 30, 36-38.

Brown, P. I., Hughes, M. G., \& Tong, R. J. (2008). The Effect of Warm-Up on High Intensity, Intermittent Running Using Non-Motorized Treadmill Ergometry. Journal of Strength and Conditioning Research, 22, 801-808. http://dx.doi.org/10.1519/JSC.0b013e31816a5775

Burkett, L. N., Phillips, W. T., \& Ziuraitis, J. (2005). The Best Warm-Up for the Vertical Jump in College-Age Athletic Men. Journal of Strength and Conditioning Research, 19, 673-676.

Chamari, K., Hachana, Y., Ahmed, Y. B., Galy, O., Sghaier, F., Chatard, J. C., Hue, O., \& Wisloff, U. (2004). Field and Laboratory Testing in Young Elite Soccer Players. British Journal of Sports Medicine, 38, 191-196. http://dx.doi.org/10.1136/bjsm.2002.004374

Ekblom, B. (1986). Applied Physiology of Soccer. Sports Medicine, 3, 50-60. http://dx.doi.org/10.2165/00007256-198603010-00005

Faigenbaum, A. D., Bellucci, M., Bernieri, A., Bakker, B., \& Hoorens, K. (2005). Acute Effects of Different Warm-Up Protocols on Fitness Performance in Children. Journal of Strength and Conditioning Research, 19, 376-381.

Faigenbaum, A. D., McFarland, J. E., Schwerdtman, J. A., Ratamess, N. A., Kang, J, \& Hoffman, J. R. (2006). Dynamic Warm-Up Protocols, with and without a Weighted Vest, and Fitness Performance in High School Female Athletes. Journal of Athletic Training, 41, 357-363.

Fitzsimons, M., Dawson, B., Ward, D., \& Wikinson, A. (1993). Cycling and Running Tests of Repeated Sprint Ability. Australian Journal of Science and Medicine in Sport, 25, 82-87.

Fradkin, A. (2002). Effects of a Warm-Up Program on Club Head Speed in Male Golfers. Master's Thesis, Melbourne: Deakin University.

Fradkin, A. J., Zazryn, T. R., \& Smoliga, J. M. (2010). Effects of Warming-UP on Physical Performance: A Systematic Review with Meta-Analysis. Journal of Strength \& Conditioning Research, 24, 140-148. http://d.x.doi.org/10.1519/JSC.0b013e3181c643a0

Gelen, E. (2010). Acute Effects of Different Warm-Up Methods on Sprint, Slalom Dribbling, and Penalty Kick Performance in Soccer Players. Journal of Strength \& Conditioning Research, 24, 950-956. http://dx.doi.org/10.1519/JSC.0b013e3181cb703f

Gourgoulis, V., Aggeloussis, N., Kasimatis, P., Mavromatis, G., \& Garas, G. (2003). Effect of Sub Maximal Half-Squats Warm-Up Program on Vertical Jumping Ability. Journal of Strength \& Conditioning Research.

Gracielle, V. R., dos Santos, R. R., \& Gonçalves, A. (2007). The Effect of Stretching on Muscle Strength: A Short Review of Possible Causes. Revista Brasileira de Cineantropometria \& Desempenho Humano.

Hedrick, A. (1992). Physiological Responses to Warm-Up. Journal of Strength \& Conditioning Research, 14, $25-27$.

Holt, B. W., \& Lambourne, K. (2008). The Impact of Different Warm-Up Protocols on Vertical Jump Performance in Male Collegiate Athletes. Journal of Strength \& Conditioning Research, 22, 226-229.

http://dx.doi.org/10.1519/JSC.0b013e31815f9d6a

Impellizzeri, F. M., Rampinini, E., Castagna, C., Bishop, D., Ferrari Bravo, D., Tibaudi, A., \& Wisloff, U. (2008). Validity of a Repeated-Sprint Test for Football. International Journal of Sports Medicine, 29, 899-905. http://dx.doi.org/10.1055/s-2008-1038491

Little, T., \& Williams, A. G. (2006). Effects of Differential Stretching Protocols during Warm-Ups on High-Speed Motor Capacities in Professional Soccer Players. Journal of Strength \& Conditioning Research, 20, 203-207.

Rampinini, E., Sassi, A., Morelli, Z., Mazzoni, S., Fanchini, M., \& Coutts, A. J. (2009). Repeated Sprint Ability in Professional and Amateur Soccer Players. Applied Physiology, Nutrition, and Metabolism, 34, 1048-1054. http://dx.doi.org/10.1139/H09-111

Richards, D. K. (1968). A Two-Factor Theory of the Warm-Up Effect in Jumping Performance. Res Q, 39, 668-673. 
Saez Saez de Villarreal, E., Gonzalez-Badillo, J. J., \& Izquierdo, M. (2007). Optimal Warm-Up Stimuli of Muscle Activation to Enhance Short and Long-Term Acute Jumping Performance. European Journal of Applied Physiology, 100, 393-401. http://dx.doi.org/10.1007/s00421-007-0440-9

Sale, D. G. (2002). Postactivation Potentiation: Role in Human Performance. Exercise \& Sport Sciences Reviews, 30, 138143. http://dx.doi.org/10.1097/00003677-200207000-00008

Sayers, A. L., Farley, R. S., Fuller, D. K., Jubenville, C. B., \& Caputo, J. L. (2008). The Effect of Static Stretching on Phases of Sprint Performance in Elite Soccer Players. Journal of Strength \& Conditioning Research, 22, 1416-1421. http://dx.doi.org/10.1519/JSC.0b013e318181a450

Spencer, M., Bishop, D., Dawson, B., \& Goodman, C. (2005). Physiological and Metabolic Responses of Repeated-Sprint Activities. Sports Medicine, 35, 1025-1044. http://dx.doi.org/10.2165/00007256-200535120-00003

Spencer, M., Fitzsimons, M., Dawson, B., Bishop, D., \& Goodman, C. (2006). Reliability of a Repeated-Sprint Test for Field-Hockey. Journal of Sports Science and Medicine, 9, 181-184. http://dx.doi.org/10.1016/j.jsams.2005.05.001

Stolen, T., Chamari, K., Castagna, C., \& Wisloff, U. (2005). Physiology of Soccer: An Update. Sports Medicine, 35, 501536. http://dx.doi.org/10.2165/00007256-200535060-00004

Wisloff, U., Castagna, C., Helgerud, J., Jones, R., \& Hoff, J. (2004). Strong Correlation of Maximal Squat Strength with Sprint Performance and Vertical Jump Height in Elite Soccer Players. British Journal of Sports Medicine, 38, 285-288. http://dx.doi.org/10.1136/bjsm.2002.002071

Woods, K., Bishop, P., \& Jones, E. (2007). Warm-Up and Stretching in the Prevention of Muscular Injury. Sports Medicine, 37, 1089-1099. http://dx.doi.org/10.2165/00007256-200737120-00006

Young, W. B., \& Behm, D. (2003). Effects of Running, Static Stretching and Practice Jumps on Explosive Force Production and Jumping Performance. Journal of Sports Medicine and Physical Fitness, 43, 21-27. 
Scientific Research Publishing (SCIRP) is one of the largest Open Access journal publishers. It is currently publishing more than 200 open access, online, peer-reviewed journals covering a wide range of academic disciplines. SCIRP serves the worldwide academic communities and contributes to the progress and application of science with its publication.

Other selected journals from SCIRP are listed as below. Submit your manuscript to us via either submit@scirp.org or Online Submission Portal.
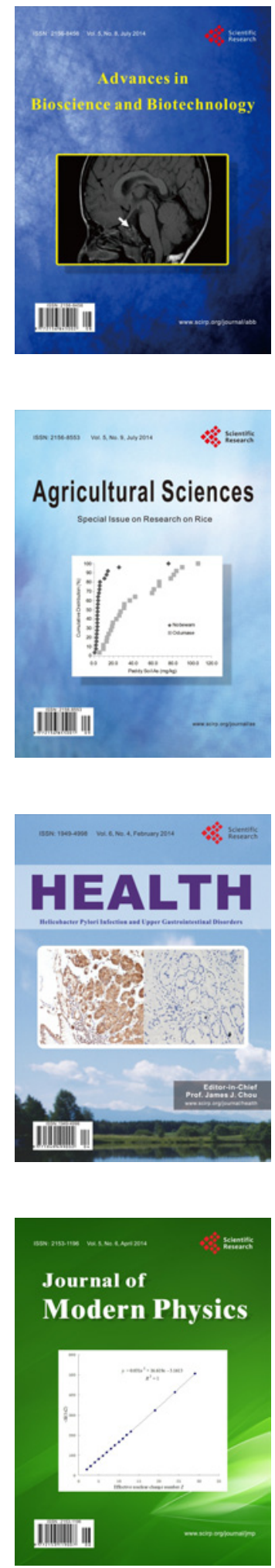
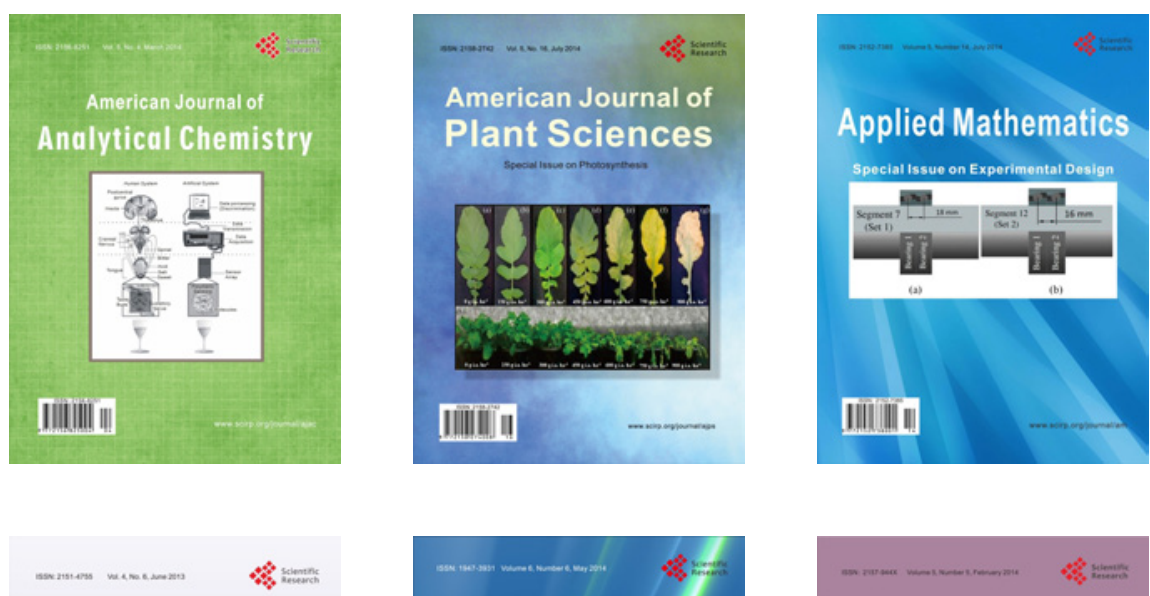

Creative Education
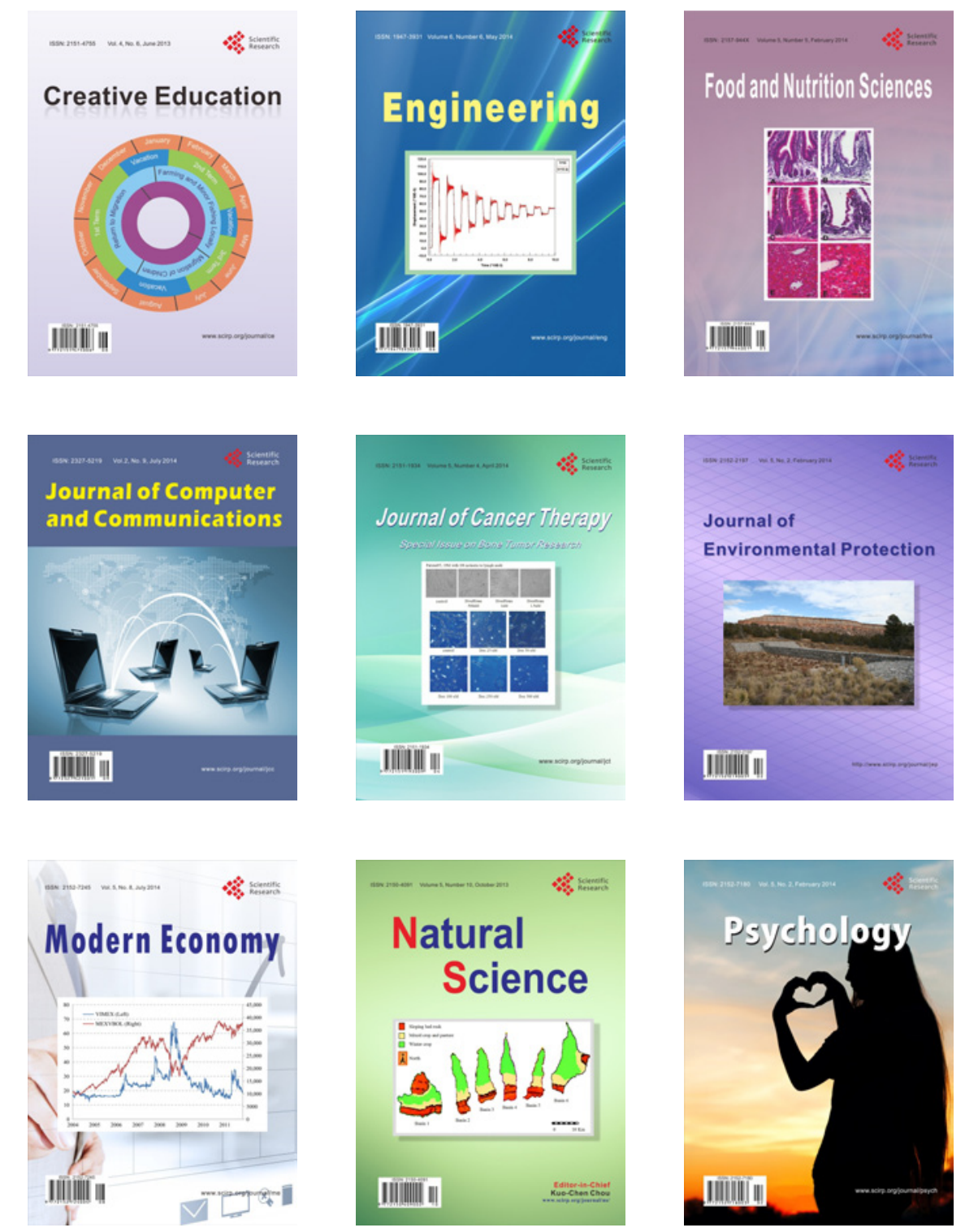